Internist 2021 · 62:337-340

https://doi.org/10.1007/s00108-021-00985-3

Angenommen: 5. Februar 2021

Online publiziert: 9. Februar 2021

(c) Springer Medizin Verlag GmbH, ein Teil von Springer Nature 2021

\section{Redaktion}

C. Bokemeyer, Hamburg

M. Hallek, Köln

C. Jacobshagen, Karlsruhe

W. Lehmacher, Köln

U. Müller-Ladner, Bad Nauheim

H. Wedemeyer, Hannover

M. Wehling, Mannheim

\section{Originalliteratur}

Baden LR, El Sahly HM, Essink B et al for the COVE Study Group (2020) Efficacy and safety of the mRNA-1273 SARS-CoV-2 vaccine. N Engl J Med. https://doi.org/10.1056/ NEJMoa2035389

Sadoff J, Le Gars M, Shukarv G et al (2021) Interim results of a phase 1-2a trial of Ad26.COV2.S covid-19 vaccine. N Engl J Med. https://doi. org/10.1056/NEJMoa2034201

Nachdem in der Januarausgabe ein erster Beitrag zur Impfstoffentwicklung für die Prävention der „coronavirus disease 2019“ (COVID-19; https://link. springer.com/article/10.1007/s00108-

020-00937-3; [1]) publiziert wurde, werden wir im Folgenden zwei weitere Impfstoffe vorstellen, den mRNA-Impfstoff mRNA-1273 (Moderna ${ }^{1}$ ) und den rekombinanten Adenovirusimpfstoff Ad26.COV2.S (Janssen ${ }^{2}$ ). Der Ad26Vektor wurde bereits für einen Ebolaimpfstoff eingesetzt, der von der Europäischen Arzneimittel-Agentur (EMA) zugelassen worden ist, sowie für Impfstoffkandidaten gegen „respiratory syncytial virus“ (RSV), „human immuno-

\footnotetext{
1 Bedingte Zulassung für die Europäische Union am 06.01.2021 erteilt.

2 "Rolling-Review-Verfahren“ bei der Europäischen Arzneimittel-Agentur (EMA) Anfang Dezember 2020 initiiert, Rekrutierung für PhaseIII-Studie (ENSEMBLE) Mitte Dezember 2020 beendet, Zulassungsantrag für Februar 2021 erwartet.
}

\author{
M. Augustin ${ }^{1} \cdot$ M. Hallek' $\cdot$ S. Nitschmann ${ }^{2}$ \\ ${ }^{1}$ Klinik I für Innere Medizin, Universitätsklinikum Köln (AöR), Köln, Deutschland \\ ${ }^{2}$ Lippetal, Deutschland
}

\title{
Impfstoffentwicklung zur Prävention von COVID-19 - Teil 2
}

deficiency virus“ (HIV) und Zika-Virus; es werden ihm eine hohe Sicherheit und Immunogenität bescheinigt [2].

Ziel der im Folgenden diskutierten Studien war es, die Wirksamkeit einer Impfung von Probanden vor einer COVID-19-Infektion zu verifizieren.

\section{Zusammenfassung der Studien}

\section{S. Nitschmann}

Lippetal, Deutschland

COVE Study Group - mRNA-1273SARS-CoV-2-Vakzine

\section{Studiendesign}

Laufende einfachblinde, kontrollierte, randomisierte Multicenterimpfstudie an 99 Zentren in den USA mit einem lipidnanopartikelformulierten nukleosidmodifizierten RNA-Impfstoff, der für ein Severe-acute-respiratory-syndrome-coronavirus-2(SARS-CoV-2)Spike-Protein in voller Länge codiert, mit einer durchschnittlichen Nachuntersuchungszeit von 64 Tagen

\section{Einschlusskriterien}

- Über 18-jährige Probanden mit erhöhtem Risiko für eine SARS-CoV2-Infektion oder deren Komplikationen, ohne bisher bekannte SARSCoV-2-Infektion

\section{Endpunkte Primär.}

- Nachweis einer symptomatischen COVID-19-Infektion mehr als 14 Tage nach der zweiten Impfung bei zu Studienbeginn seronegativen Probanden:

- 2 der folgenden Symptome: Fieber $\geq 38^{\circ} \mathrm{C}$, Schüttelfrost, Myalgie, Kopfschmerz, Halsschmerzen oder Geruchs-/Geschmacksverlust oder

- mindestens ein respiratorisches Symptom (Husten, Atemnot, Anzeichen einer Lungenentzündung) und mindestens ein positiver Nasenabstrich, Atemtest, Speichelprobe oder

- positiver Polymerase-Kettenreaktions(PCR)-Test

Sekundär.

- Effektivität bei der Verhinderung einer schweren COVID-19-Infektion

\section{Take home message}

Der mRNA-Impfstoff mRNA-1273 von Moderna stellt mit einer Effektivität von 94,1\% einen sicheren und effektiven Impfstoff zur Prävention von COVID19 dar. Ad26.COV2.S von Janssen Vaccines \& Prevention B.V. stellt einen neuen sicheren und immunogenen Vektorimpfstoffkandidaten dar, dessen Wirksamkeit und Dosierungsschema (einzeitig vs. zweizeitig) derzeit in einer Phase-III-Studie untersucht werden. Zur endgültigen Beurteilung der Wirksamkeit sind diese Ergebnisse abzuwarten. 
(Atemfrequenz $\geq 30 / \mathrm{min}$, Herzfrequenz $\geq 125 / \mathrm{min}$, Sauerstoffsättigung $\leq 93 \%$ bei Raumluft, respiratorisches Versagen, „acute respiratory distress syndrome", Schock, klinisch gravierendes akutes Nierenbzw. Leberversagen, neurologische Dysfunktion, Intensivpflichtigkeit, Tod)

- Effektivität nach nur einer Impfdosis - Impfstoffsicherheit

\section{Methodik}

Die 30.420 Probanden wurden, stratifiziert nach Alter und Risiko für einen komplikationsträchtigen COVID-19Verlauf, 1:1 auf zwei Impfungen mit $100 \mu \mathrm{g}$ mRNA-1273-SARS-CoV-2-Vakzine bzw. Placebo in 28-tägigem Abstand randomisiert, wobei die erste Impfung zwischen dem 27.07. und dem 23.10.2020 verabreicht wurde.

Vor den Impfungen wurden bei den Probanden ein SARS-CoV-2-Antikörpertest und ein nasopharyngealer Abstrich (PCR) durchgeführt. Infizierte Probanden wurden 14 Tage lang oder bis zur Besserung der Symptome täglich zur Beurteilung der Schwere des Verlaufs untersucht. Bei Probanden, die Symptome einer COVID-19-Infektion aufwiesen, wurde ein nasopharyngealer PCR-Test durchgeführt und ein serologischer SARS-CoV-2-Nachweis angestrebt.

Subgruppenanalysen nach Geschlecht, Alter, ethnischer Gruppe, Risikofaktoren für einen schweren Verlauf sowie Risiko für SARS-CoV-2-Infektion wurden durchgeführt.

\section{Ergebnisse}

In die Intention-to-treat-Analyse gingen 14.550 bzw. 14.598 Probanden ein, in die Per-protocol-Analyse 14.134 bzw. 14.073 Probanden. Bei diesen wurde die Effektivitätsanalyse durchgeführt. Eine serologisch oder virologisch nachgewiesene SARS-CoV-2-Infektion wiesen bei Studienbeginn 2,2\% aller Probanden auf (337 in der Placebo- und 343 in der mRNA-1273-Gruppe).

Mehr als $96 \%$ aller Probanden erhielten beide Injektionen. Bei 114 Patienten wurde in der PCR vor der zweiten Impfung SARS-CoV-2 nachgewiesen: bei 69 in der Placebo- und 45 in der mRNA1273-Gruppe.

Das Durchschnittsalter betrug 51,4 Jahre, etwas mehr als die Hälfte der Probanden waren Männer (52,7\% Männer vs. $47,3 \%$ Frauen). 58,6\% der Probanden waren zwischen 18 und 65 Jahre alt und wiesen keine Risikofaktoren für eine schwere COVID-19-Infektion auf, 16,7\% waren zwischen 18 und 65 Jahre alt und wiesen Risikofaktoren auf. Die übrigen 24,8\% der Probanden waren 65 Jahre oder älter. Deutliche Unterschiede gab es auch bezüglich der Ethnien: 79,2\% Weiße, 10,2\% Schwarze, 4,6\% Asiaten wurden in die Studie eingeschlossen.

Primärer Endpunkt Eine symptomatische COVID-19-Infektion trat bei 185 der Placebo- und bei 11 der mRNA1273-Patienten auf (56,5/1000 Personenjahre bzw. 3,3/1000 Personenjahre). Dies entspricht einer Impfeffektivität von $94,1 \%(p<0,001)$.

Sekundärer Endpunkt Insgesamt traten 30 schwere COVID-19-Verläufe auf: alle in der Placebogruppe.

Im Zeitraum von 2 Wochen nach der ersten Impfung bis zum 25.11.2020 kam es bei 225 der Placebo- und bei 11 der mRNA-1273-Patienten zu einer symptomatischen COVID-19-Infektion, was einer Impfeffektivität von 95,2\% entspricht. Die Impfeffektivität war in allen Subgruppen vergleichbar. Eine asymptomatische SARS-CoV-2-Infektion (positiver PCR-Test) wurde bei 39 primär SARS-CoV-2-negativen Placeboprobanden und bei 15 primär SARSCoV-2-negativen mRNA-1273-Probanden $(0,3 \%$ vs. $0,1 \%)$ detektiert.

Unerwünschte lokale Impfnebenwirkungen traten bei $84,2 \%$ der mRNA1273-Probanden und bei $19,8 \%$ der Placeboprobanden nach der ersten Impfung und bei $88,6 \%$ bzw. 18,8\% nach der zweiten Impfung auf, wobei die häufigste unerwünschte Wirkung „Schmerzen an der Injektionsstelle“ (86\%) waren. Mehr als 8 Tage nach der ersten Impfung traten bei $0,8 \%$, nach der zweiten bei $0,2 \%$ unerwünschte Wirkungen auf, am häufigsten Erythem und lokale Verhärtungen, die sich binnen der nächsten 4-5 Tage zurückbildeten.
Unerwünschte systemische Impfnebenwirkungen traten bei $54,9 \%$ der mRNA-1273-Probanden und bei 42,2\% der Placeboprobanden nach der ersten Impfung und bei $79,4 \%$ bzw. $36,5 \%$ nach der zweiten Impfung auf. Diese dauerten durchschnittlich 2,6 bzw. nach der zweiten Impfung 3,1 Tage an und traten am häufigsten bei jüngeren $\mathrm{Pa}$ tienten auf. Schwere Nebenwirkungen waren bei den mRNA-1273-Probanden häufiger: $0,5 \%$ vs. $0,2 \%$.

\section{Sadoff J et al. - Ad26.COV2.S- COVID-19-Vakzine}

\section{Studiendesign}

Laufende einfachblinde, kontrollierte, randomisierte, 5-armige Multicenterimpfstudie (Phase I/II) mit einem rekombinanten replikationsinkompetenten Adenovirusserotyp 26 (Ad26), der für das SARS-CoV-2-Spike-Protein in voller Länge codiert, an 12 Zentren in Belgien und den USA

\section{Einschlusskriterien}

- Gesunde, zufällig ausgewählte Probanden zwischen 18 und 55 Jahren (Kohorte 1)

- Gesunde, zufällig ausgewählte Probanden $\geq 65$ Jahre (Kohorte 3 )

\section{Endpunkte Primär.}

- Sicherheit und Effektivität einzelner Impfstoffdosispläne

\section{Sekundär.}

- Humorale und zelluläre Immunität gegen SARS-CoV-2

\section{Methodik}

In die hier vorgestellte Zwischenanalyse gehen die Daten der Patienten aus Kohorte 1 und 3 bis zum 30.10.2020 ein. Vom 22.07.2020 bis zum 07.08.2020 wurden 593 Probanden für Kohorte 1 gescreent. Von diesen erhielten 402 die erste Dosis Ad26.COV2.S. Eine zweite Impfdosis nach 56 Tagen erhielten diese Probanden bis zum 07.11.2020.

Vom 03.08. bis 24.08.2020 wurden 660 Probanden für Kohorte 3 gescreent, von denen 403 die erste Dosis Ad26.COV2.S erhielten. 
Es wurden zwei unterschiedliche Dosierungen in unterschiedlichen Kombinationen getestet: $5 \cdot 10^{10}$ Viruspartikel/ml (niedrig dosiert [LD]) vs. $1 \cdot 10^{11}$ Viruspartikel/ml (hoch dosiert [HD]). Die Probanden der Kohorte 1 und 3 wurden 1:1:1:1:1 randomisiert auf - erste Dosis LD, zweite Dosis LD,

- erste Dosis LD, zweite Dosis Placebo,

- erste Dosis HD, zweite Dosis HD,

- erste Dosis HD, zweite Dosis Placebo,

- erste Dosis Placebo, zweite Dosis

Placebo,

Follow-up-Untersuchungen zur Analyse der unterwünschten Impfwirkungen erfolgten 7, 28 und 71 Tage nach der Impfung jeder Kohorte. Mittels „enzymelinked immunosorbent assay" (ELISA) wurden SARS-CoV-2-Antikörper bei Studieneinschluss sowie nach 15, 29, 57 und 71 Tagen gemessen, wobei Seropositivität als ein Titer $>50,3 \mathrm{EU} / \mathrm{ml}$ definiert wurde. Spezifische T-Zell-Antworten wurden bei Studieneinschluss sowie nach 15 Tagen mittels intrazellulärer Zytokinfärbung gemessen. In CD4+-T-Zellen wurde die Expression von Interferon- $\gamma$ und/oder Interleukin-2 analysiert.

\section{Ergebnisse}

Es werden die Daten der Kohorte 1 nach der zweiten Impfung und die Daten der Kohorte 3 nach der ersten Impfung vorgestellt.

Das Durchschnittsalter in Kohorte 1 betrug 35,4 Jahre, $52 \%$ waren Frauen, SARS-CoV-2-seropositiv bei Studienbeginn waren 7 Probanden; in Kohorte 3 (69,8 Jahre und 50\% Frauen) 4 Probanden.

Sicherheit Die unterwünschten Impfnebenwirkungen waren zumeist leichte $\mathrm{Ne}$ benwirkungen: Fieber, Müdigkeit, Kopfschmerzen, Myalgien und Schmerzen an der Injektionsstelle waren die häufigsten. Lokale Nebenwirkungen wurden in der Kohorte 1 von $64 \%$ der LD-, $78 \%$ der HD- und 9\% der Placebopatienten angegeben; in der Kohorte 3 von $41 \%, 42 \%$ und $14 \%$.

Bei den über 65-Jährigen traten unerwünschte systemische Nebenwirkungen seltener auf als bei jüngeren Probanden; ebenso seltener bei Probanden, die eine niedrigere Dosis bekommen hatten, als bei den Probanden mit höherer Dosis: in Kohorte 1 bei $65 \%$ der LD-, 84 \% der HDund $26 \%$ der Placebopatienten; in Kohorte 3 bei $46 \%, 55 \%$ und $23 \%$. Schwere systemische Nebenwirkungen ereigneten sich in Kohorte 1 bei $9 \%$ der LD-, $20 \%$ der HD- und $0 \%$ der Placebopatienten; in Kohorte 3 bei $1 \%$, $2 \%$ und $0 \%$. Von keinem der Probanden wurde die Studie aufgrund der unerwünschten Wirkungen abgebrochen.

\section{Humorale und zelluläre Immunität}

Neutralisierende Antikörper wurden an Tag 29 nach der ersten Impfdosis bei $>90 \%$ aller Probanden nachgewiesen mit einer weiteren Titererhöhung bei allen Probanden an Tag 57. Bis Tag 71 blieben die Titer stabil. Die zweite Impfdosis führte zu einer 2,6- bis 2,9-fachen Titererhöhung.

In Kohorte 1 wurde eine Serokonversion, außer in der Hochdosis-PlaceboGruppe (97\%), bei $100 \%$ der Probanden nach der ersten Impfung erreicht; nach der zweiten Impfung in allen Verumgruppen $100 \%$. In Kohorte 3 wurde nach 15 Tagen bei $75 \%$ bzw. $77 \%$ der Probanden eine Serokonversion erreicht, an Tag 29 bei $96 \%$.

Eine Serokonversion für neutralisierende Antikörper (SARS-CoV-2-Titer) wurde in Kohorte 1 am Tag 29 je nach Dosierungsgruppe bei 88-96\% der Probanden erreicht, am Tag 57 bei $100 \%$; in Kohorte 3 bei 84 und $91 \%$ sowie an Tag 57 bei 88 und $96 \%$.

Die durch den Impfstoff ausgelöste Reaktion in spezifischen CD4+-T-Zellen an Tag 14 wurde bei $76-83 \%$ der Kohorte-1- und bei $60-67 \%$ der Kohorte-3Probanden nachgewiesen.

\section{Kommentar}

\section{Augustin, Prof. Dr. M. Hallek}

Klinik I für Innere Medizin, Universitätsklinikum Köln (AöR), Köln, Deutschland

\section{mRNA-1273-SARS-CoV-2-Vakzine}

Nach BNT162b2 von BioNTech/Pfizer [1] ist mRNA-1273 von Moderna der zweite Messenger-Ribonukleinsäure
(mRNA)-Impfstoff, der von der EMA in der Europäischen Union zugelassen wurde (Stand: 26.01.2021). Denn auch hier liegt mittlerweile eine methodisch korrekte, placebokontrollierte, 1:1 randomisierte sowie verblindete klinische Phase-II/III-Studie zur Evaluation der Sicherheit und Effektivität einer zweizeitigen Verabreichung von jeweils $100 \mu \mathrm{g}$ mRNA-1273 (Intervall 28 Tage) vor. So konnte an insgesamt 30.420 Probanden eine mRNA-1273-Impfeffektivität von $94,1 \%$ nachgewiesen werden. Während die Geschlechterverteilung in der Studienpopulation ausgeglichen war, gab es Unterschiede im Hinblick auf Alter und Ethnien der Studienpopulation (s. oben). Lokale und systemische unerwünschte Ereignisse („adverse events“ [AE]) waren in der Verumpopulation deutlich häufiger als im Placeboarm. Diese unerwünschten Ereignisse sind als Impfreaktion zu werten, die im Mittel am dritten bis vierten Tag sistieren. Bei lediglich $0,5 \%$ schweren unerwünschten Ereignissen konnte mRNA-1273 (Moderna) als sicher bewertet werden.

Im direkten Vergleich zu BNT162b2 (BioNTech/Pfizer), dem bereits zugelassenen mRNA-Impfstoff zur Prävention von COVID-19, besteht eine Impfdosis von mRNA-1273 (Moderna) aus 3,3-mal so viel mRNA (BNT162b2: $30 \mu \mathrm{g}$ mRNA; mRNA-1273 $100 \mu \mathrm{g}$ ). Dies könnte eine Erklärung für die häufigeren und länger andauernden Impfreaktionen nach mRNA-1273 vs. BNT162b2 sein (Häufigkeit: mRNA-1273: 84,2\%, BNT162b2: 27\%; Dauer: mRNA-1273: 2,6-3,2 Tage, BNT162b2: 1-2 Tage). Ob die höhere mRNA-Menge Auswirkungen auf das Erreichen einer sterilen Immunität oder die Dauer des immunologischen Schutzes hat, sollte Gegenstand zukünftiger Studien werden.

Zusammengefasst stellt der zweizeitige mRNA-Impfstoff mRNA-1273 von Moderna mit einer Effektivität von 94,1 \% einen sicheren und effektiven Impfstoff zur Prävention von COVID-19 dar - die Zulassungskriterien der Food and Drug Administration (FDA) für die Zulassung von Impfstoffen zur Prävention von COVID-19 (Effektivität $>50 \%$; [3]) wurden deutlich übertroffen. 
Aufgrund unterschiedlicher Mutationen in der Rezeptorbindungsstelle des Spike-Proteins ist es zum Auftreten von mutierten Virusvarianten von SARSCoV-2 aus Großbritannien (B.1.1.7; [4]), Südafrika (B.1.351; [5]) und Brasilien (P.1; [6]) gekommen. Erste Untersuchungen zeigen, dass impfvermittelte Antikörper vor allem die Variante B.1.1.7 neutralisieren können. Die Wirksamkeit der Antikörper gegen Variante B.1.351 scheint nach abgeschlossener Impfung sukzessiv abzunehmen. Moderna hat aus diesem Grund bereits einen adaptierten Folgeimpfstoff entwickelt, dessen klinische Prüfung noch aussteht.

\section{Ad26.COV2.S-COVID-19-Vakzine}

Auch in der Klasse der Vektorimpfstoffe, in der AZD122 (AstraZeneca/ University of Oxford) von der EMA am 29.01.2021 zugelassen wurde [1], gibt es mit Ad26.COV2.S. von Janssen Vaccines \& Prevention B.V. einen weiteren potenziellen Kandidaten, der sich aktuell im Rolling-Review-Verfahren der EMA befindet. Mit der hier vorliegenden placebokontrollierten und randomisierten Phase-I/IIa-Studie konnten die Sicherheit und Immunogenität von Ad26.COV2.S bewertet werden. In zwei gleich großen Kohorten mit unterschiedlichen Altersverteilungen wurde der auf nichtreplizierenden adenoviralen Vektoren beruhende Impfstoff in

- ein- und zweizeitiger Verabreichung sowie

- unterschiedlichen Dosiskombinationen (HD und LD)

getestet.

Lokale und systemische unerwünschte Ereignisse wurden häufiger in der Hochdosis- als in der Niedrigdosis- oder Placebogruppe verzeichnet (lokale AE: HD $78 \%$ vs. LD $64 \%$ vs. Placebo $9 \%$; systemische AE: HD $84 \%$ vs. LD $65 \%$ vs. Placebo $26 \%(n=21))$. Es traten keine schweren unerwünschten Ereignisse auf, die $\mathrm{zu}$ einem Studienabbruch geführt hätten. Sowohl nach zweizeitiger Impfung der jüngeren Kohorte 1 als auch nach der einzeitigen Impfung der älteren
Kohorte 3 konnte Ad26.COV.S in dieser Phase-I/IIa-Studie neutralisierende Antikörper produzieren sowie spezifische T-Zell-Antworten generieren. Obwohl bei der zweizeitigen Verabreichung von Ad26.COV2.S die Immunogenität insgesamt höher war, könnte die Einmalgabe von Ad26.COV2.S ausreichen, um eine effektive Immunogenität $\mathrm{zu}$ generieren. Dies und die offene Frage nach der Wirksamkeit von Ad26.COV2.S ist Gegenstand der laufenden multizentrischen, placebokontrollierten, doppelblinden und randomisierten Phase-IIIStudie von Janssen (ENSEMBLE), deren Ergebnisse abzuwarten sind.

\section{Korrespondenzadresse}

\section{Dr. med. M. Augustin}

Klinik I für Innere Medizin, Universitätsklinikum Köln (AöR)

Kerpener Str. 62, 50937 Köln, Deutschland

max.augustin@uk-koeln.de

\section{Einhaltung ethischer Richtlinien}

Interessenkonflikt. M. Augustin, M. Hallek und S. Nitschmann geben an, dass kein Interessenkonflikt besteht.

Für diesen Beitrag wurden von den Autoren keine Studien an Menschen oder Tieren durchgeführt. Für die aufgeführten Studien gelten die jeweils dort angegebenen ethischen Richtlinien.

\section{Literatur}

1. Augustin M, Hallek M, Nitschmann S (2021) Development of vaccines for prevention of COVID19. Internist 62(1):106-110

2. Anywaine $Z$, Whitworth $H$, Kaleebu $P$ et al (2019) Safety and immunogenicity of a 2dose heterologous vaccination regimen with Ad26.ZEBOV and MVA-BN-Filo Ebola vaccines: 12 month data from a phase 1 randomized clinical trial in Uganda and Tanzania. JInfect Dis 220:46-56

3. Food and Drug Administration (2020) Guidance for industry: emergency use authorization for vaccines to prevent COVID-19. October 2020. https://www.fda.gov/media/142749/download (p. 15).Zugegriffen: 26. Jan. 2021

4. Volz E et al (2021) Transmission of SARS-CoV-2 lineage B.1.1.7 in England: insights from linking epidemiological and genetic data. medRxiv. https://doi.org/10.1101/2020.12.30.20249034

5. Tegally $\mathrm{H}$ et al (2020) Emergence and rapid spread of a new severe acute respiratory syndromerelated coronavirus 2 (SARS-CoV-2) lineage with multiple spike mutations in South Africa. medRxiv. https://doi.org/10.1101/2020.12.21.20248640
6. TeFaria NR, Claro IM, Candido D et al. Genomic characterisation of an emergent SARS-CoV2 lineage in Manaus: preliminary findings. Virological. https://virological.org/t/genomiccharacterisation-of-an-emergent-sars-cov-2lineage-in-manaus-preliminary-findings/586. Zugegriffen: 26. Jan. 2021 